\title{
The Adsorption of Per- and Polyfluoroalkyl Substances (PFASs) onto Ferrihydrite Is Governed by Surface Charge
}

\author{
Hugo Campos-Pereira,* Dan B. Kleja, Carin Sjöstedt, Lutz Ahrens, Wantana Klysubun,
} and Jon Petter Gustafsson

Cite This: Environ. Sci. Technol. 2020, 54, 15722-15730

Read Online

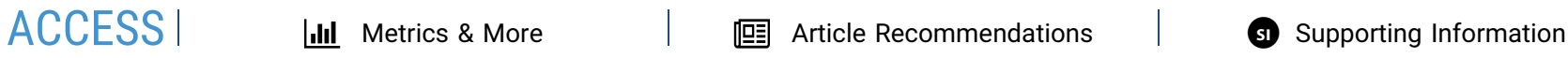

ABSTRACT: An improved quantitative and qualitative understanding of the interaction of per- and polyfluoroalkyl substances (PFASs) and short-range ordered Fe (hydr)oxides is crucial for environmental risk assessment in environments low in natural organic matter. Here, we present data on the $\mathrm{pH}$-dependent sorption behavior of 12 PFASs onto ferrihydrite. The nature of the binding mechanisms was investigated by sulfur K-edge X-ray absorption near-edge structure (XANES) spectroscopy and by phosphate competition experiments. Sulfur K-edge XANES spectroscopy showed that the sulfur atom of the head group of the sulfonated PFASs retained an oxidation state of $+\mathrm{V}$ after adsorption. Furthermore, the XANES spectra did not indicate any involvement of inner-sphere surface complexes in the sorption

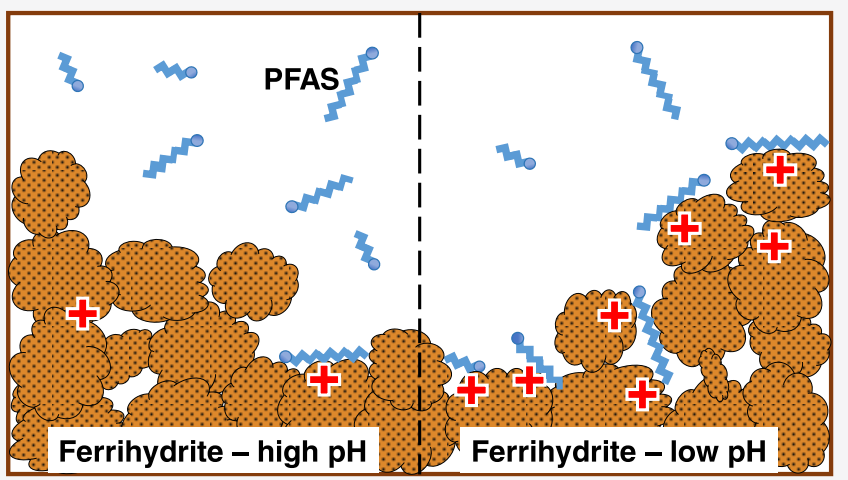
process. Adsorption was inversely related to $\mathrm{pH}(p<0.05)$ for all PFASs (i.e., $\mathrm{C}_{3}-\mathrm{C}_{5}$ and $\mathrm{C}_{7}-\mathrm{C}_{9}$ perfluorocarboxylates, $\mathrm{C}_{4}, \mathrm{C}_{6}$, and $\mathrm{C}_{8}$ perfluorosulfonates, perfluorooctane sulfonamide, and 6:2 and 8:2 fluorotelomer sulfonates). This was attributed to the $\mathrm{pH}$ dependent charge of the ferrihydrite surface, as reflected in the decrease of surface $\zeta$-potential with increasing $\mathrm{pH}$. The importance of surface charge for PFAS adsorption was further corroborated by the observation that the adsorption of PFASs decreased upon phosphate adsorption in a way that was consistent with the decrease in ferrihydrite $\zeta$-potential. The results show that ferrihydrite can be an important sorbent for PFASs with six or more perfluorinated carbons in acid environments $(\mathrm{pH} \leq 5)$, particularly when phosphate and other competitors are present in relatively low concentrations.

\section{INTRODUCTION}

Accurate prediction of the binding of per- and polyfluoroalkyl substances (PFASs) in soil is essential for environmental risk assessment. However, information on PFAS binding to poorly crystalline iron (hydr)oxides such as ferrihydrite is scarce up to date, despite that these phases are known to be important for contaminant and trace element soil retention ${ }^{1-3}$ due to their large surface area ${ }^{4,5}$ and high reactivity. ${ }^{6}$ The poorly crystalline $\mathrm{Fe}$ (hydr)oxide ferrihydrite has a high abundance in many young soils, particularly in cool or temperate climates with high moisture and occurrence of $\mathrm{Si}$ and/or organic matter. ${ }^{7}$ For example, ferrihydrite is present in concentrations up to $2 \%$ in the B horizon of Swedish Podzols. ${ }^{8,9}$ Because of its high reactivity and high surface area, ranging from 250 to $1260 \mathrm{~m}^{2}$ $\mathrm{g}^{-1}$, ferrihydrite is an important adsorbent in many soils, particularly for anions. ${ }^{6}$ Subsoils are conceptually interesting environments as they delay the transport of solutes between the soil surface horizon and ground- and surface waters. As PFASs such as perfluorooctane sulfonate (PFOS) and perfluorooctane carboxylate (PFOA) are common groundwater contaminants, ${ }^{10-12}$ it is of importance for environmental risk assessment to gain more knowledge on PFAS sorption behavior to subsoil materials, especially as regards sorption to poorly crystalline $\mathrm{Fe}$ (hydr)oxides like ferrihydrite.

Up to date, studies on PFAS binding to mineral surfaces have mostly focused on the sorption properties of crystalline minerals such as alumina $\left(\mathrm{Al}_{2} \mathrm{O}_{3}\right)$, silica $\left(\mathrm{SiO}_{2}\right),{ }^{13-15}$ hematite $\left(\alpha\right.$ - $\left.\mathrm{Fe}_{2} \mathrm{O}_{3}\right),{ }^{16,17}$ boehmite $(\gamma$-AlOOH $),{ }^{18,19}$ and goethite $(\alpha$ $\mathrm{FeOOH}),{ }^{14,20,21}$ and of minerals that are typical of aquatic sediments such as, for example, the silicates kaolinite and montmorillonite. ${ }^{16,22,23}$ Besides, many studies up to date have been limited to include one single PFAS, i.e., PFOS. As regards binding mechanisms responsible for adsorption onto mineral surfaces, previous works have often emphasized the outersphere or electrostatic contributions ${ }^{13,20,21}$ over those of hydrophobic interactions or specific (inner-sphere) complexes. However, using attenuated total reflection infrared spectros-

Received: March 16, 2020

Revised: October 30, 2020

Accepted: November 8, 2020

Published: November 27, 2020

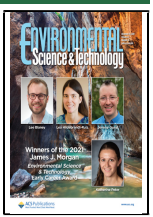


copy, specific binding of PFOA has been reported to take place on hematite ${ }^{17}$ and on ferrihydrite ${ }^{24}$ under acidic conditions, although the results of Zhang et al. ${ }^{25}$ suggest outer-sphere complexes on oxidized zero-valent iron. For PFOS, innersphere complexes on Fe oxides have not been reported. Instead outer-sphere complexes and/or electrostatic attractions may predominate. ${ }^{17,25}$ The ferrihydrite surface is positively charged at $\mathrm{pH}$ values below its point of zero charge (PZC), which is $\mathrm{pH}$ $\sim 8.1$ for pure ferrihydrite. ${ }^{4,26}$ Hence, it may be expected that the adsorption of anionic PFASs by ferrihydrite is stronger at low $\mathrm{pH}$. Furthermore, it may be hypothesized that PFAS adsorption onto ferrihydrite decreases in the presence of other adsorbed anions at the ferrihydrite surface, as specific binding of such species lowers the positive surface charge. For example, phosphate $\left(\mathrm{PO}_{4}{ }^{3-}\right)$ is a common oxyanion in the environment, which forms strong surface complexes; hence, phosphate may affect PFAS binding both by direct competition for sites and by modifying the surface charge. ${ }^{27}$

PFASs such as PFOS and perfluorooctane sulfonamide (FOSA) contain a sulfur (S) atom as part of their head group. The chemistry and speciation of sulfur-containing compounds may be investigated using spectroscopic methods such as, e.g., $\mathrm{X}$-ray absorption near-edge structure (XANES) and extended X-ray absorption fine structure (EXAFS) spectroscopy. ${ }^{28-33}$ The absorption edge, corresponding to the excitation of an inner-shell electron, exhibits several identifiable features that change depending on the chemical environment of the sulfur atom. For example, inner-sphere complexes involving $\mathrm{Fe}$ give rise to a so-called pre-edge due to orbital hybridization, as, for example, observed for the inner-sphere complexes of sulfate on ferrihydrite. ${ }^{32,33}$ Hence, in the present study, we employed $S$ K-edge XANES spectroscopy to investigate the bonding characteristics of sulfonated PFASs onto ferrihydrite.

To our knowledge, this is the first study to investigate the adsorption behavior of a range of different PFASs onto ferrihydrite $(\mathrm{Fh})$. The specific objectives were to (1) investigate the $\mathrm{pH}$-dependent $\mathrm{Fh}$ binding of PFASs in the absence and presence of phosphate $\left(\mathrm{PO}_{4}{ }^{3-}\right)$ as a competing anion and (2) reveal the adsorption mechanism of selected sulfonated PFASs onto Fh using S K-edge XANES spectroscopy in combination with $\zeta$-potential measurements and results from batch experiments.

\section{MATERIALS AND METHODS}

Target PFASs. Twelve PFASs (standards purchased from Sigma-Aldrich) were analyzed, i.e., $\mathrm{C}_{3}-\mathrm{C}_{5}$ and $\mathrm{C}_{7}-\mathrm{C}_{9}$ perfluoroalkyl carboxylates (PFCAs), $\mathrm{C}_{4}, \mathrm{C}_{6}$, and $\mathrm{C}_{8}$ perfluoroalkyl sulfonates (PFSAs), perfluorooctane sulfonamide (FOSA), and 6:2 and 8:2 fluorotelomer sulfonates (6:2 and 8:2 FTSA). For quantification as well as for quality assurance and control ( $\mathrm{QA} / \mathrm{QC})$, eight mass-labeled internal standards (ISs) (i.e., ${ }^{13} \mathrm{C}_{4}$-PFBA, ${ }^{13} \mathrm{C}_{2}$-PFHxA, ${ }^{13} \mathrm{C}_{4}$-PFOA, ${ }^{13} \mathrm{C}_{5}$-PFNA, ${ }^{13} \mathrm{C}_{2}$-PFDA, ${ }^{18} \mathrm{O}_{2}$-PFHxS, ${ }^{13} \mathrm{C}_{4}$-PFOS, ${ }^{13} \mathrm{C}_{8}$-FOSA, purities $>99 \%$, Wellington Laboratories, Guelph, ON) were also included. For details on the included PFASs and their internal standards, see Table S1 in the Supporting Information.

Ferrihydrite and Al Hydroxide Preparation. 2-Line ferrihydrite was synthesized using the method of Swedlund and Webster $^{34}$ and Schwertmann and Cornell ${ }^{35}$ with minor modifications. In short, a solution containing $36 \mathrm{mmol}$ $\mathrm{Fe}\left(\mathrm{NO}_{3}\right)_{3} \mathrm{~L}^{-1}$ was brought to $\mathrm{pH} 8.0$ through dropwise addition of freshly prepared sodium hydroxide $(4 \mathrm{~mol} \mathrm{NaOH}$ $\left.\mathrm{L}^{-1}\right)$ under magnetic stirring. The resulting suspension was left to settle and age for about $16 \mathrm{~h}$ at $20^{\circ} \mathrm{C}$ in a tightly capped high-density polyethylene (HDPE) bottle ( $500 \mathrm{~mL}$, Nalgene). Iron (hydr)oxide particles from such a suspension have previously been studied by Fe K-edge EXAFS spectroscopy,36 and were confirmed to be poorly crystalline 2-line ferrihydrite. After synthesis, the Fh suspension was back-titrated to $\mathrm{pH} 4.6$ with dropwise addition of $0.1 \mathrm{~mol}$ nitric acid $\left(\mathrm{HNO}_{3}\right) \mathrm{L}^{-1}$ under magnetic stirring and stirred for some additional $30 \mathrm{~min}$ before the start of the batch experiments to remove $\mathrm{CO}_{2}$. For the preparation of $\mathrm{Fh}$ subjected to $\mathrm{S}$ K-edge XANES spectroscopy, high-purity $\mathrm{HNO}_{3}$ (purchased from SigmaAldrich) was used to minimize trace element (in particular S) contamination. The properties of the Fh synthesized in this way (e.g., surface area, aggregation state, etc.) have been described by Hiemstra. ${ }^{6,26}$ For example, the surface area for the Fh produced in our laboratory has been estimated at 650 or $611 \mathrm{~m}^{2} \mathrm{~g}^{-1}$, depending on the assumption made on the molar weight of ferrihydrite. ${ }^{2,6}$ Furthermore, for use in the spectroscopic measurements, poorly crystalline aluminum hydroxide (Alhox) was synthesized with the same method as was used for the preparation of ferrihydrite, with the only modifications being that $\mathrm{Fe}\left(\mathrm{NO}_{3}\right)_{3}$ was substituted for $\mathrm{Al}\left(\mathrm{NO}_{3}\right)_{3}$, and that the suspension was titrated to $\mathrm{pH} 7.0$ before aging and back-titrated to $\mathrm{pH} 5.0$ after aging.

X-ray Absorption Spectroscopy. For the S K-edge XANES measurements, PFASs adsorbed to Fh and Alhox were sampled in their wet paste state from sorption batch experiments. The purpose of including Alhox in these experiments was that a pre-edge peak, resulting from innersphere complexation of $\mathrm{SO}_{4}{ }^{2-}$, would be expected on $\mathrm{Fh}$, but not on Alhox. ${ }^{30,33}$ Hence, the existence of a pre-edge peak resulting from orbital hybridization on Fh could more easily be separated from other features in the $\mathrm{S}$ K-edge XANES spectrum. Spectra for adsorbed sulfate $\left(\mathrm{SO}_{4}{ }^{2-}\right)$ were also collected for comparison, as were those of pure PFAS standards and potassium sulfate $\left(\mathrm{K}_{2} \mathrm{SO}_{4}\right)$ salt in their solid states. The batch experiments were performed immediately before the spectroscopic measurements. In brief, concentrations of $200 \mu \mathrm{mol} \mathrm{L}{ }^{-1}$ of selected sulfonated PFASs (i.e., PFHxS (counterion $\mathrm{K}), \mathrm{PFOS}(\mathrm{K})$, FOSA, or sulfate (added as $\left.\mathrm{K}_{2} \mathrm{SO}_{4}\right)$ ) were equilibrated for $24 \mathrm{~h}$ with either Fh or Alhox (1 mmol $\mathrm{L}^{-1} \mathrm{Fe}$ or $\mathrm{Al}$, respectively) before phase separation. Supernatant residual sulfate and PFASs were quantified to determine adsorbed concentrations (Table S13 in the Supporting Information). Sulfate was determined with ion chromatography according to ISO 10 304-1:2009 (accredited). The S K-edge XANES spectra were collected in fluorescence mode over the energy range of $2400-2550 \mathrm{eV}$ at beamline BL8 at the Synchrotron Light Research Institute (SLRI), Nakhon Ratchasima, Thailand, ${ }^{37}$ in May 2017. Details on beamline equipment are given in Section S1.1 in the Supporting Information, and instrument settings are provided elsewhere. ${ }^{31}$ Samples were diluted with boron nitride (BN), placed into $\mathrm{Al}$ frames, and sealed with $\mathrm{X}$-ray film using sulfurfree Kapton tape (Lanmar, Inc.). Energy calibration was done using the sulfate white-line of $\mathrm{FeSO}_{4}$ at $2482.5 \mathrm{eV}^{31}$ All XANES spectra were corrected for energy shift and normalized using the Athena software ${ }^{38}$ (version 0.9.26). Multiple scans were merged and subsequently normalized to yield a K-edge intensity step of unity. This was done by subtracting linear and quadratic baseline functions over the pre-edge and normalization (post-edge) range, respectively. Relative to the whiteline position, the employed pre-edge ranges were from -70 to 


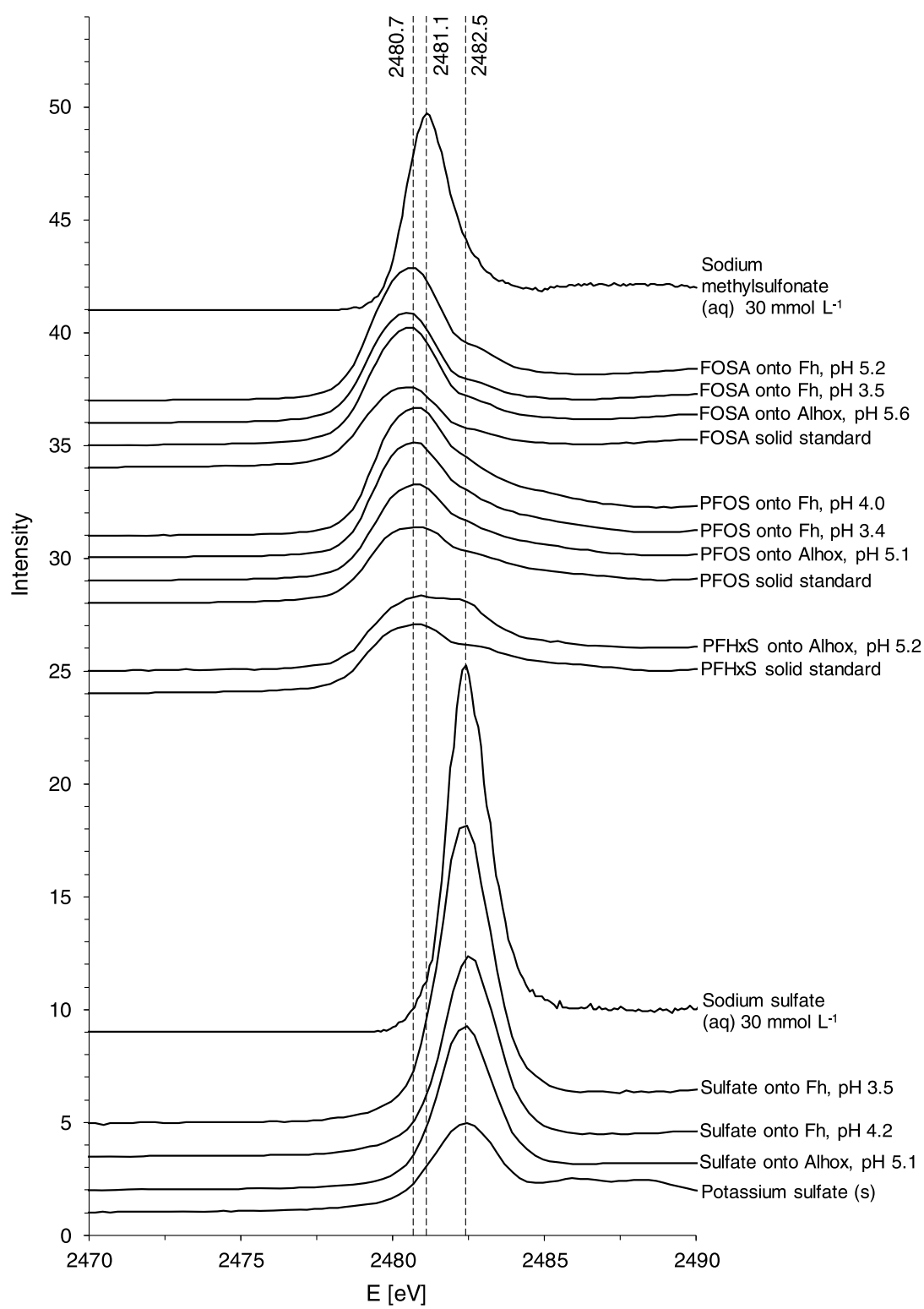

Figure 1. Stacked normalized S K-edge XANES spectra for sulfonated PFASs adsorbed to ferrihydrite (Fh) and poorly crystalline aluminum hydroxide (Alhox). Dashed white-line positions are, from lower to higher energies, those of PFOS onto Fh and Alhox, of dissolved dilute sodium methylsulfonate $\left(\mathrm{NaCH}_{3} \mathrm{SO}_{3}\right)$, and of sulfate onto $\mathrm{Fh}$ and Alhox. Spectra for sodium sulfate $\left(\mathrm{Na}_{2} \mathrm{SO}_{4}\right)$ (aq) and sodium methylsulfonate were provided by Almkvist et al., ${ }^{52}$ and their intensities were set to unity at $2490 \mathrm{eV}$ for the purpose of comparison.

$-25 \mathrm{eV}$ for PFASs and from -65 to $-25 \mathrm{eV}$ for sulfate, whereas the normalization range was from +40 to $+60 \mathrm{eV}$.

\section{BATCH EXPERIMENTS}

$\mathrm{pH}$-Dependent Binding in the Absence and Presence of Phosphate. A ferrihydrite stock suspension was mixed with Milli-Q water and $\mathrm{NaNO}_{3}$ solution in $50 \mathrm{~mL}$ reactors (polypropylene centrifuge tubes) to obtain final concentrations of $3.2 \mathrm{mmol} \mathrm{Fe} \mathrm{L}^{-1}$ as ferrihydrite and $10 \mathrm{mmol}$ nitrate $\mathrm{L}^{-1}$. Various amounts of acid $\left(\mathrm{HNO}_{3}\right)$ or base $(\mathrm{NaOH}$, prepared the same day) were then added to reach $\mathrm{pH}$ values between 4.0 and 7.6 after equilibration. Subsequently, 0 or $200 \mu \mathrm{mol} \mathrm{L}{ }^{-1}$ phosphate $\left(\right.$ as $\left.\mathrm{NaH}_{2} \mathrm{PO}_{4}\right)$ was added. For details, see Table S2 in the Supporting Information. Finally, triplicate sets of centrifuge tubes were spiked with $20 \mu \mathrm{L}$ of a stock mixture of 12 PFASs dissolved in methanol (analytical grade, Merck) so that the initial aqueous concentrations of individual PFASs ranged from $1.6 \mathrm{nmol} \mathrm{L}^{-1}$ (PFDA) to $5.5 \mathrm{nmol} \mathrm{L}^{-1}$ (PFBS) (Table S3 in the Supporting Information). The additions were sufficiently low to ensure that sorption occurred in the linear range (cf. below). Duplicate negative controls were included for quality assurance and control. Suspensions were end-overend shaken $\left(45 \mathrm{rpm}, 21^{\circ} \mathrm{C}\right)$ for $24 \mathrm{~h}$ prior to centrifugation at $\sim 2000 \mathrm{~g}$ for about $30 \mathrm{~min}$. The $\mathrm{pH}$ was measured in the supernatant of subsamples using a Red $\mathrm{Rod} \mathrm{Ag} / \mathrm{AgCl}$ electrode (Radiometer Analytical SAS). The ferrihydrite $\zeta$-potential was measured at $21{ }^{\circ} \mathrm{C}$ (Zetasizer Nano-ZS, Malvern) on nonspiked suspensions ( $\mathrm{pH}$ 3.9-9.5) having the same solution chemistry and phosphate additions as samples in the sorption experiment (Table $\mathrm{S} 10$ in the Supporting Information). Aqueous phosphate and $\mathrm{Fe}(0.45 \mu \mathrm{m}$ filtration, Sartorius Minisart hydrophilic regenerated cellulose, $\varnothing 25 \mathrm{~mm})$ were analyzed using inductively coupled plasma-optical emission spectroscopy (ICP-OES) (Table S4 in the Supporting 
Information). Iron was quantified ( $\mathrm{LoQ}=40 \mu \mathrm{g} \mathrm{L}^{-1}$ ) to ensure efficient filter retention of ferrihydrite particles and to check that no iron dissolution of significance occurred in the investigated $\mathrm{pH}$ range.

Sorption Isotherms. Varying volumes of either PFOA, PFOS, or FOSA dissolved in methanol $\left(20 \mathrm{mmol} \mathrm{L}^{-1}\right)$ were added to $\mathrm{Fh}$ suspensions $\left(10 \mathrm{mmol} \mathrm{Fe} \mathrm{L}^{-1}\right)$ prior to equilibration for $24 \mathrm{~h}$ using end-over-end shaking. Initial additions of the individual PFASs were chosen so that aqueous concentrations after equilibration spanned approximately 3 orders of magnitude (i.e., ca. 1-700 nmol PFOS $\mathrm{L}^{-1}, 1-2000$ nmol PFOA L $\mathrm{L}^{-1}, 0.3-1000 \mathrm{nmol}$ FOSA $\left.\mathrm{L}^{-1}\right)$. After centrifugation, $\mathrm{pH}$ was measured in supernatants of sample aliquots, and was confirmed to be $4.5 \pm 0.1$.

PFAS Analysis. For quantification of aqueous PFAS concentrations, $5 \mathrm{ng}$ of each of the mass-labeled ISs was added to sample aliquots prior to gentle filtration $(0.45 \mu \mathrm{m}$, Sartorius Minisart hydrophilic regenerated cellulose $\left.{ }^{39,40}\right)$. The PFAS concentrations were measured by the means of IS isotope dilution using direct injection in a Dionex UltiMate 3000 ultrahigh-performance liquid chromatography (UPLC) system (Thermo Fisher Scientific, Massachusetts) coupled to a triple-quadrupole tandem mass spectrometer (MS/MS) (TSQ Quantiva, Thermo Fisher Scientific, Massachusetts). Sorption to ferrihydrite was calculated as the difference between added and dissolved PFASs, with the exception of that of substances that showed recoveries below $90 \%$ in the positive controls, for which losses to reactor walls were also taken into account (see below).

Quality Assurance and Control. All experiments used Milli-Q water, which was filtered through a cartridge containing powdered activated carbon (PAC) (Milli-Q LCPak, Merck) to minimize contamination. For the same purpose, no fluorinated materials (e.g., tetrafluoroethylene, Teflon) were used in the experiments. All PFASs in the negative controls $(n=2)$ were below the limit of quantification (LoQ), as defined as the lowest quantifiable calibration point (i.e., the lowest calibration point having a response factor within $\pm 30 \%$ of the average response factor of the calibration curve). The method recovery was calculated from the losses of ISs due to sample preparation and matrix effects, as determined by comparison with the calibration curve, and was, for individual PFASs, on average $86 \pm 3 \%$. The percent standard deviation of individual aqueous PFAS concentrations was, on average, $7 \%$ in the $\mathrm{pH}$-dependent sorption experiment $(n=3)$, and $5 \%$ as regards the sorption isotherms $(n=2)$. The fraction of spiked co-solvent methanol in the isotherm and the $\mathrm{pH}$ dependent sorption experiments was $<0.3 \%(\mathrm{v} / \mathrm{v})$, and thus the effect of methanol on PFAS partitioning could be considered negligible. ${ }^{41}$

Aqueous recoveries in positive controls without ferrihydrite ( $n=4,10 \mathrm{mM} \mathrm{NaNO}$, pH 5.8, equilibration $24 \mathrm{~h}$ ) were typically higher than $90 \%$ of the total added amount of PFASs (sum of amounts in the solution phase and in $\mathrm{MeOH}$ extract of the empty control reactor). For substances that showed control recoveries below 90\%, i.e., PFNA (86\%), PFDA (87\%), and FOSA (80\%), the calculated $\mathrm{pH}$-dependent sorption to ferrihydrite was corrected for losses to the reactor walls using a three-compartment equilibrium-based approach (Section S1.3 and Figure S1 in the Supporting Information). However, as reactor losses of organic chemicals (including PFOA) generally decrease with increasing aqueous concentrations, $^{39,42,43}$ the sorption isotherm of FOSA was not recalculated according to the above method, as we in this experiment employed significantly higher concentrations compared to those used in the $\mathrm{pH}$-dependent sorption test and in the positive controls (i.e., most nominal isotherm additions were 50-2500 times larger).

\section{RESULTS AND DISCUSSION}

X-ray Absorption Spectroscopy. As shown in Figure 1, the sulfur K-edge white-line positions for adsorbed sulfonated PFASs (e.g., that of PFOS; $2480.7 \mathrm{eV}$ ) were close to that of dissolved dilute methylsulfonate $(2481.1 \mathrm{eV})$, in which the sulfur atom has a formal and electronic oxidation state of $+\mathrm{V}$. All of these white-line positions were also similar to those of the pure, solid PFAS standards (i.e., 2480.4-2480.9 eV; Table S13 in the Supporting Information). As the electronic oxidation state of $S$ increases linearly with the white-line position relative to that of elemental (0) sulfur, ${ }^{28,44-49}$ it can be concluded that the sulfur atom in all measured PFASs (i.e., PFOS, FOSA, PFHxS) retained an oxidation state of $+\mathrm{V}$ after the adsorption of these PFASs onto both ferrihydrite and poorly crystalline $\mathrm{Al}$ hydroxide. In agreement with previous studies, sulfate onto Fh showed a pre-edge (Figure S6 in the Supporting Information), which reflects the involvement of inner-sphere complexes. ${ }^{30,33,50,51}$ A similar pre-edge was not observed for any of the three S-containing PFASs onto ferrihydrite, as no clear feature could be identified on the lowenergy side of the main absorption peak, and as the spectra for the S-containing PFASs onto the Al hydroxide were identical in shape (Figures S7-S13 in the Supporting Information). Therefore, these results rule out a significant role of innersphere complexes for S-containing PFASs on ferrihydrite, in agreement with previous infrared evidence for PFOS on Fe oxides. $^{17,25}$ Still, it is possible, or even likely, that the head group can form an outer-sphere complex. ${ }^{17}$ However, such an interaction is normally weak and would imply a strong role of electrostatic interactions in the PFAS binding process.

pH-Dependent Adsorption onto Ferrihydrite. The adsorption of all 12 investigated PFASs (i.e., PFBA, PFPeA, PFHxA, PFOA, PFNA, PFDA, PFBS, PFHxS, PFOS, FOSA, 6:2 FTSA, 8:2 FTSA) was inversely related to $\mathrm{pH}(p<0.05)$ (Figure 2 and Tables S4-S8 in the Supporting Information). This was attributed to the $\mathrm{pH}$-dependent charge of the ferrihydrite surface, i.e., the lower the $\mathrm{pH}$ value, the larger the positive surface net charge (Figure 3 ) and thus the larger the attraction between the ferrihydrite surface and the negatively charged PFAS anions. At $\mathrm{pH}$ values between 4 and 5 , the $\zeta$ potential was $>27 \mathrm{mV}$, which decreased to nearly zero at $\mathrm{pH}$ 7.7, in agreement with the $\mathrm{PZC}$ of $\mathrm{pH} \sim 8.1$ reported for 2-line ferrihydrite. $^{26}$ At $\mathrm{pH}$ values $\leq 5$, there was a stronger adsorption of long-chained PFASs (i.e., PFOA, PFNA, PFDA, PFHxS, PFOS, FOSA, 6:2 FTSA, 8:2 FTSA) compared to that of the short-chained PFASs (i.e., PFBA, PFPeA, PFHxA, PFBS) (Figures 2 and S2-S4 and Table S7 in the Supporting Information). For example, at $\mathrm{pH} 4$, the sorption percentage of the long-chained PFASs was between $60 \%$ (PFOA) and 100\% (PFDA), whereas it ranged from $31 \%$ (PFPeA) to $43 \%$ (PFHxA) for the short-chained PFASs. However, at $\mathrm{pH}$ values higher than 5 , the overall sorption was low, and there was no evident difference in the degree of adsorption between long-chained and short-chained PFASs (Figures 2 and S2-S4 and Table S7 in the Supporting Information). This indicates that to achieve $>50 \%$ sorption to ferrihydrite, a strongly positively charged surface is required $(\zeta$ - 


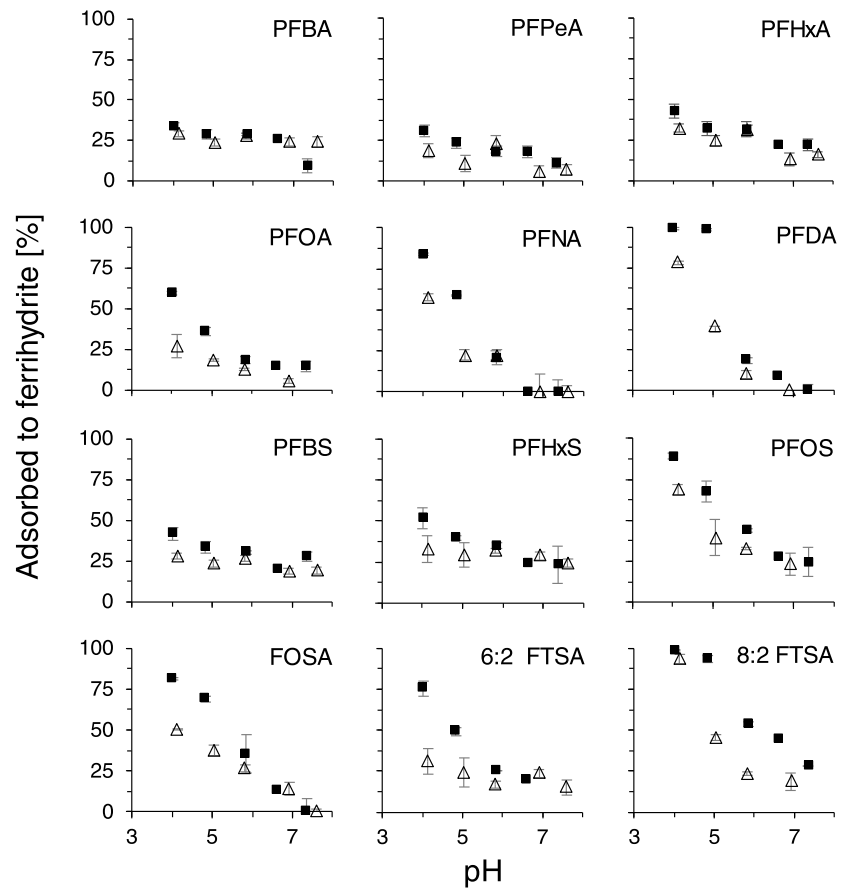

- $3.2 \mathrm{mmol} \mathrm{Fe} \mathrm{L}{ }^{-1} \Delta 3.2 \mathrm{mmol} \mathrm{Fe} \mathrm{L}^{-1}+200 \mu \mathrm{mol}$ phosphate $\mathrm{L}^{-1}$

Figure 2. Effect of $\mathrm{pH}$ on PFAS adsorption onto ferrihydrite in the absence and presence of phosphate. PFAS additions ranged from 1.6 nmol L ${ }^{-1}$ (PFDA) to $5.5 \mathrm{nmol} \mathrm{L}^{-1}$ (PFBS). The error bars represent the standard error of the mean $(n=3)$.

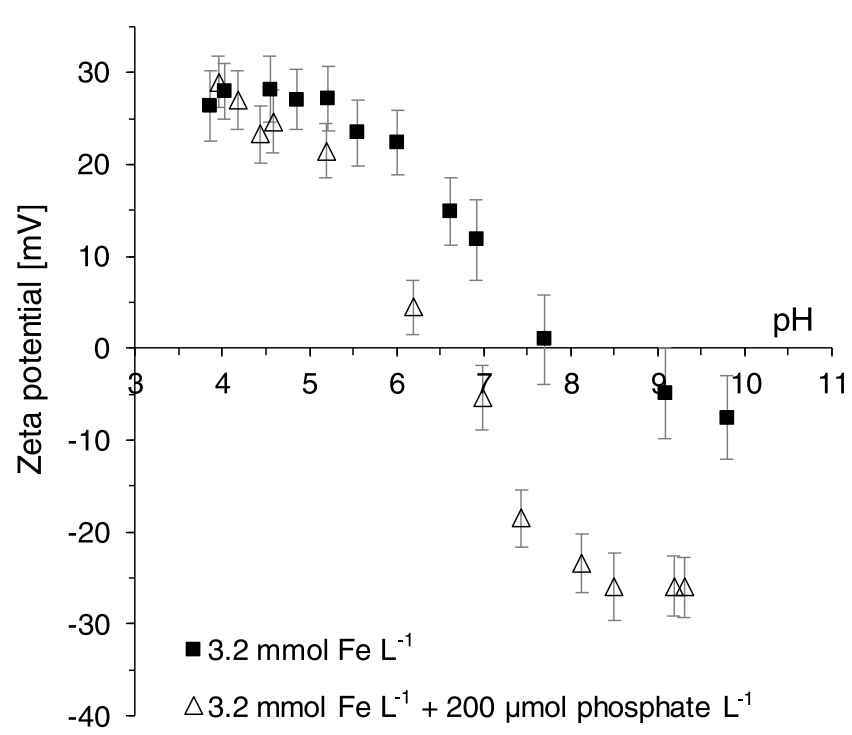

Figure 3. Ferrihydrite $\zeta$-potential as a function of $\mathrm{pH}$ and added phosphate. The error bars represent the standard deviation within each measurement.

potential $>+20$ to $25 \mathrm{mV}$, Figure 3), and in addition, the PFAS molecule needs to be of sufficient chain length in terms of its number of perfluorinated carbons (i.e., $\mathrm{C}_{6}$ or longer).

At low $\mathrm{pH}$, the binding of the long-chained PFASs was significant despite the fact that the sulfonate and carboxylate head groups are weakly charged and likely not able to compete strongly with the electrolyte anions $\left(0.01 \mathrm{M} \mathrm{NO}_{3}^{-}\right)$in the studied systems, if only electrostatic interactions of the head group were involved. Hence, such a mechanism is not sufficient to explain PFAS binding. Further, the observation that long-chained PFASs are sorbed more strongly than shortchained PFASs to ferrihydrite strongly suggests that the role of specific interactions (i.e., as inner-sphere or outer-sphere complexes), although they may exist, is rather small, despite earlier research showing surface complex formation between the head group of a PFAS (PFOS and PFOA) and Fe oxide. $^{17,24,25}$

Instead, the stronger adsorption of the long-chained PFASs is consistent with a substantial additional contribution of the weakly negatively charged fluorine moieties to the overall electrostatic interaction, as suggested by Johnson et al. ${ }^{21}$ and Xiao et al. ${ }^{23}$ Although the charge excess for dissolved PFOS has been reported to be only around -0.1 to -0.2 per fluorine atom, ${ }^{23,53}$ the electron density should be sufficient to induce hydrogen bonding between the fluorine moieties and positively charged $-\mathrm{OH}_{2}{ }^{1 / 2+}$ or $-\mathrm{O}_{3} \mathrm{H}^{1 / 2+}$ groups of ferrihydrite, which may act as proton donors. The probable significance of a strong involvement of the tail in the sorption process is underlined also by the strong sorption of FOSA at low $\mathrm{pH}$, as the head group of FOSA (i.e., $\mathrm{SO}_{2} \mathrm{NH}_{2}$ ) is not charged under low- $\mathrm{pH}$ conditions $\left(\mathrm{p} K_{\mathrm{a}}=6.24,{ }^{54} 6.52^{55}\right)$. The differences between different PFASs may be explained by multiple F' $\mathrm{HO}$ sorptive contacts for long-chained PFASs, which would stabilize sorption. A related mechanism has earlier been suggested for PAH sorption on goethite, based on DFT calculations. ${ }^{56}$ For a short-chained PFAS such as PFBS, there are fewer fluorine atoms available for binding, which makes sorption rather inefficient also at low $\mathrm{pH}$.

Effects of Phosphate and Surface Charge. In general, the adsorption of PFASs onto ferrihydrite decreased with the addition of phosphate (Figure 2). The decreased PFAS binding can be related to the $\zeta$-potential, which also decreased with increasing $\mathrm{pH}$ at $\mathrm{pH}>5$ (Figure 3 ). With the exception of PFBA, the decrease in adsorption of all investigated PFASs upon the addition of phosphate anions was significantly $(p<$ 0.05 ) related to the decrease in positive charge on the ferrihydrite surface ( $\zeta$-potential) due to the presence of adsorbed phosphate (Figures 4 and S5, linear regression given in Table S9 in the Supporting Information). The strong relationship with the $\zeta$-potential for the sorption of individual PFASs in both phosphate-containing and phosphate-free systems provides further support to the idea forwarded in the previous section, i.e., that electrostatic interactions are important for PFAS sorption. However, for two of the weakest binding PFASs, i.e., PFBS and PFBA, the relationship with the $\zeta$-potential was less convincing (Figure 4 ), and in the case of PFBA statistically nonsignificant (Table S9 in the Supporting Information). Whether this is due to uncertainties in the analytical measurements or reflects the presence of an additional weak sorption mechanism (e.g., oleophobic) cannot be determined from the current data.

Sorption Isotherms. As evident from Figure 5, the sorption isotherms of PFOS and PFOA onto ferrihydrite were both nonlinear (Tables S11 and S12 in the Supporting Information), and for both isotherms, the Langmuir equation provided a better fit compared to the Freundlich equation (Table S12 in the Supporting Information). Hence, the isotherms may be interpreted as being consistent with adsorption in the form of a monolayer, as also suggested by Tang et al. ${ }^{14}$ and Johnson et al. ${ }^{21}$ for nonlinear adsorption of PFOS onto goethite. In this study, the Langmuir maximum 


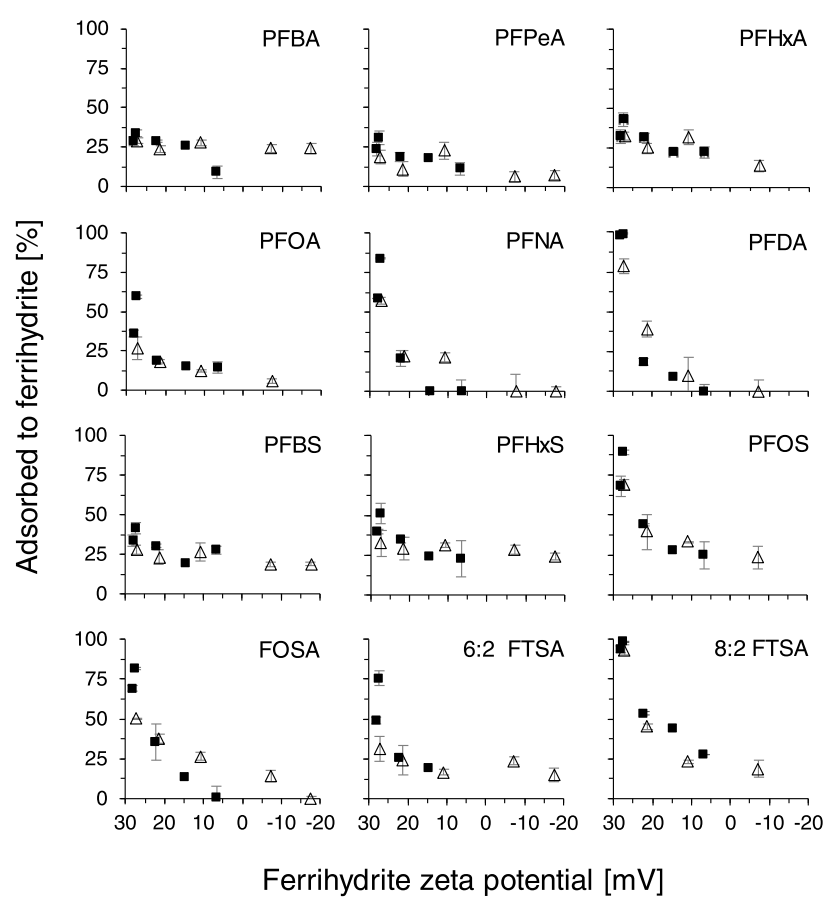

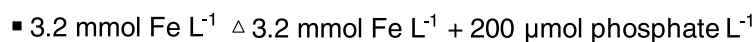

Figure 4. PFAS adsorption onto ferrihydrite as a function of $\zeta$ potential in the absence and presence of added phosphate. $\zeta$-potential values on the horizontal axis are given in reverse order, to reflect the negative relationship with the $\mathrm{pH}$ value.

ferrihydrite surface coverage $Q_{\max }$ was calculated to be 180 and $160 \mu \mathrm{mol} \mathrm{mol}^{-1} \mathrm{Fe}$, equivalent to 1.6 and $1.4 \mu \mathrm{g} \mathrm{m}^{-2}$ for PFOS and PFOA, respectively, when a surface area of $650 \mathrm{~m}^{2} \mathrm{~g}^{-1}$ for ferrihydrite is used. These values are low in comparison to the calculated net positive charge of the ferrihydrite of $\sim 10 \mathrm{mmol}$ $\mathrm{mol}^{-1} \mathrm{Fe}$, as calculated by the model of Tiberg et al. ${ }^{2}$ for the experimental conditions given in Figure 5, but for PFOS, they are reasonably close to the Langmuir maxima previously given for goethite, i.e., 1.2 and $2.4 \mu \mathrm{g} \mathrm{m}^{-2}$ by Johnson et al. ${ }^{21}$ and Tang et al., ${ }^{14}$ respectively. The $K_{\mathrm{d}}$ value (i.e., the solidsolution distribution coefficient) for adsorption of PFOS onto ferrihydrite at $\mathrm{pH} 4.5$ is about $3300 \mathrm{~L} \mathrm{~kg}^{-1} \mathrm{Fe}$ at an aqueous equilibrium concentration of $0.75 \mu \mathrm{mol}$ PFOS $\mathrm{L}^{-1}$. This is essentially the same value as that obtained for "nanosized
$\mathrm{Fe}_{2} \mathrm{O}_{3}$ " ( $K_{\mathrm{d}}$ recalculated from the work of $\mathrm{Lu}$ et al. $\left.{ }^{57}\right)$, despite the higher $\mathrm{pH}$ (7.0) of the $\mathrm{Fe}_{2} \mathrm{O}_{3}$ system. Still, the estimated $Q_{\max }$ values obtained in our work are surprisingly low compared to what would be expected for inorganic ions. This suggests that not all of the charged sites were readily accessible to the PFASs, for steric reasons or because of the aggregated and microporous structure of our ferrihydrite, which may have prevented the PFASs from binding to a large part of the charged ferrihydrite structure.

The sorption isotherm of FOSA onto ferrihydrite was relatively linear up to an approximate aqueous equilibrium concentration of $0.65 \mu \mathrm{mol} \mathrm{L} \mathrm{L}^{-1}$ (Figure 5). In the isotherm experiments, the overall order of sorption affinity to ferrihydrite was PFOS $>$ FOSA $\approx$ PFOA. Thus, the presence of a negatively charged head group $\left(\mathrm{SO}_{3}{ }^{-}\right.$in $\left.\mathrm{PFOS}\right)$ was generally observed to favor sorption compared to the presence of an uncharged functional group $\left(\mathrm{SO}_{2} \mathrm{NH}_{2}\right.$ (FOSA)), suggesting that for PFOS, both the charged head group and the fluorine moieties were important for sorption. However, at the highest aqueous concentration of the respective isotherm, the adsorption of FOSA was significantly larger compared to that of PFOS and PFOA. A possible explanation for the higher adsorption of FOSA at high aqueous concentration may be that the FOSA molecules self-aggregated, i.e., formed hemimicelles, ${ }^{23,58}$ at the ferrihydrite-solution interface. For amphiphilic compounds, it has been estimated that hemimicelles may be formed at mineral surfaces when the aqueous concentration exceeds between 0.1 and $1 \%$ of the critical micelle concentration (CMC). ${ }^{41}$ While no values for the CMC of FOSA were found in the literature, the CMC of PFOS has been reported to lie in the range of $6.3-9 \mathrm{mmol} \mathrm{L}^{-1}$. ${ }^{59-61}$ However, given the stronger hydrophobic character of the neutral FOSA molecule compared to that of the charged PFOS anion, one may assume that FOSA would exhibit a somewhat lower CMC in relation to PFOS. If so, this could make FOSA hemimicelle formation on ferrihydrite possible at aqueous concentrations of approximately $1 \mu \mathrm{mol} \mathrm{L}{ }^{-1}$, consistent with the observed isothermal sorption of the substance.

Environmental Implications. The results presented here indicate that at low $\mathrm{pH}$, ferrihydrite and other poorly crystalline $\mathrm{Fe}$ and $\mathrm{Al}$ (hydr)oxides could contribute significantly to binding of PFASs in soils. For example, $\log K_{d}$ values $\left(/ \mathrm{L} \mathrm{kg}^{-1} \mathrm{Fe}\right)$ for PFOS and PFOA at $\mathrm{pH} 5$ were approximately 4.1 and 3.5 , respectively, in the absence of phosphate. Hence, partitioning onto pure ferrihydrite was $1.4 \pm 0.1 \log$ units
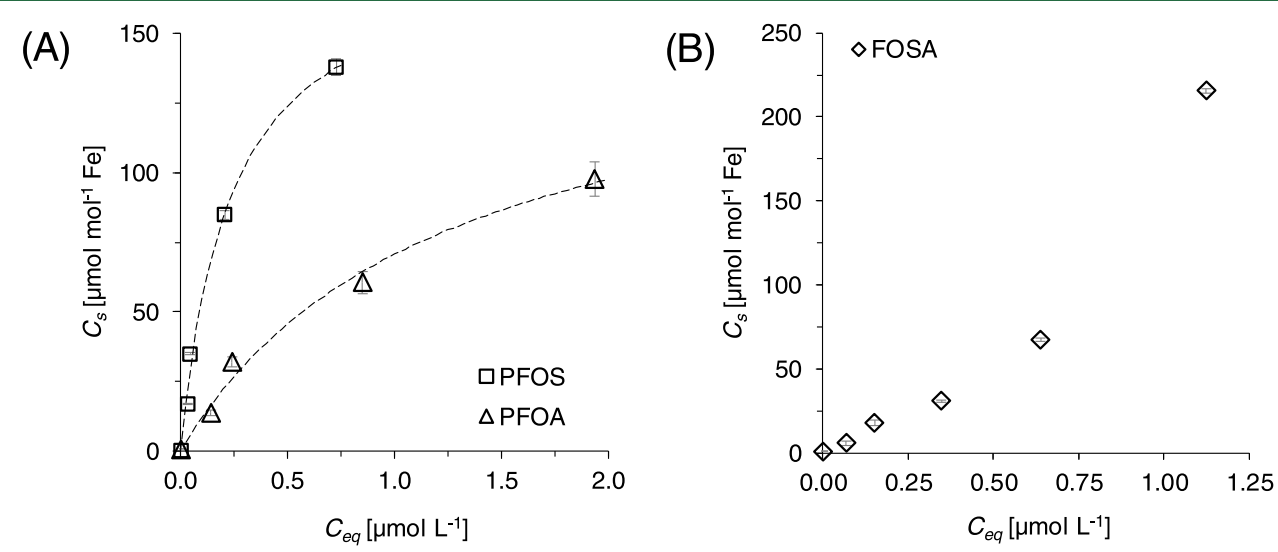

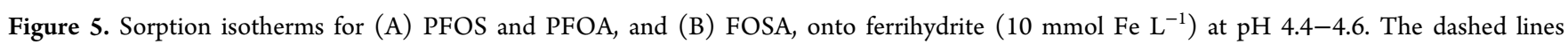
represent the fitted Langmuir isotherms. 
larger than the corresponding organic carbon-normalized sorption $\left(\log K_{\mathrm{OC}}\left(/ \mathrm{L} \mathrm{kg}^{-1} \mathrm{C}\right)\right)$ commonly found for midrange soil and sediment concentrations, i.e., $\sim 2.8$ and $\sim 2.0$ log units for PFOS and PFOA, respectively. ${ }^{62-68}$

However, it needs to be acknowledged that the properties of pure ferrihydrite systems will differ from those found in the field. Perhaps most crucially, the net positive surface charge of $\mathrm{Fe}$ and $\mathrm{Al}$ (hydr)oxides in soils and sediments is expected to be lower than for the pure minerals, due to the presence of adsorbed phosphate and organic matter. For example, the PZC of naturally occurring ferrihydrite has been reported to be between $\mathrm{pH} 5.3$ to 7.5 with emphasis on the lower values, ${ }^{69,70}$ i.e., significantly lower than that of laboratory-derived ferrihydrite. Consequently, under field conditions, the contribution of $\mathrm{Fe}$ and $\mathrm{Al}$ (hydr)oxides to PFAS binding is likely to be smaller than under laboratory conditions with isolated, pure mineral phases. The surface charge of natural ferrihydrite is critical not least because PFAS sorption is predominantly electrostatic in nature, and that surface complexes with the head group, if they are formed, are weak: if stronger inner-sphere complexes had formed, the PFAS binding would have been stronger and less sensitive to the surface charge. Although some studies do indicate a role of $\mathrm{Fe}$ and $\mathrm{Al}$ (hydr)oxides for PFAS sorption, for example, in tropical soils, ${ }^{71}$ additional research is needed to more accurately determine the relative roles of organic matter and (hydr)oxide surfaces.

In summary, the above findings allow us to conclude that $\mathrm{Fe}$ oxides such as ferrihydrite could be important for PFAS sorption in acidic environments ( $\mathrm{pH} 5$ or lower), especially if the content of organic matter and phosphate (that decrease the surface charge) is low, and if the PFAS in question is of sufficient chain length, $\mathrm{C}_{6}$ or longer.

\section{ASSOCIATED CONTENT}

\section{(s) Supporting Information}

The Supporting Information is available free of charge at https://pubs.acs.org/doi/10.1021/acs.est.0c01646.

Beamline equipment for $S$ K-edge XANES measurements; details on PFAS analytical method; positive control recoveries and method to correct for losses to reactors; PFAS native and internal standards; chemical recipe for $\mathrm{pH}$-dependent sorption experiment; $\mathrm{pH}$ dependent sorption data; linear regressions; $\zeta$-potentials; phosphate adsorption onto ferrihydrite; isotherm sorption data and its derived parameters; details on samples measured with S K-edge XANES spectroscopy and their spectra; and normalized spectra, and spectra derivatives, for adsorbed individual PFASs and sulfate (PDF)

\section{AUTHOR INFORMATION}

\section{Corresponding Author}

Hugo Campos-Pereira - Department of Soil and Environment, Swedish University of Agricultural Sciences (SLU), SE-750 07 Uppsala, Sweden; 이이.org/00000002-8023-0767; Phone: +46 (0)18 671243;

Email: hugo.pereira@slu.se

\section{Authors}

Dan B. Kleja - Department of Soil and Environment, Swedish University of Agricultural Sciences (SLU), SE-750 07
Uppsala, Sweden; Swedish Geotechnical Institute (SGI), SE58193 Linköping, Sweden

Carin Sjöstedt - Department of Soil and Environment, Swedish University of Agricultural Sciences (SLU), SE-750 07 Uppsala, Sweden; ○ orcid.org/0000-0001-6490-1658

Lutz Ahrens - Department of Aquatic Sciences and Assessment, Swedish University of Agricultural Sciences (SLU), SE-750 07 Uppsala, Sweden; (1) orcid.org/00000002-5430-6764

Wantana Klysubun - Synchrotron Light Research Institute, Nakhon Ratchasima 30000, Thailand

Jon Petter Gustafsson - Department of Soil and Environment, Swedish University of Agricultural Sciences (SLU), SE-750 07 Uppsala, Sweden; Department of Sustainable Development, Environmental Science and Engineering, KTH Royal Institute of Technology, SE-100 44 Stockholm, Sweden

Complete contact information is available at: https://pubs.acs.org/10.1021/acs.est.0c01646

\section{Notes}

The authors declare no competing financial interest.

\section{ACKNOWLEDGMENTS}

This research was gratefully supported by the Swedish Research Council (Vetenskapsrådet) (grant number 201503938). The authors further acknowledge Geert Cornelis and Jani Tuoriniemi at the Swedish University of Agricultural Sciences (SLU), for initial assistance with $\zeta$-potential measurements. G. Almkvist, K. Boye, and I. Persson are acknowledged for kindly providing $S$ K-edge XANES spectra for reference. The authors thank the staff at Beamline 8 (BL8), Synchrotron Light Research Institute (Nakhon Ratchasima, Thailand), for support during the XANES data collection. The authors also thank Farid Jan (SLU) for quantification of aqueous iron and phosphate, and Vera Franke and Mattias Sörengård (SLU) for assistance with parts of the PFAS analyses.

\section{REFERENCES}

(1) Jambor, J. L.; Dutrizac, J. E. Occurrence and Constitution of Natural and Synthetic Ferrihydrite, a Widespread Iron Oxyhydroxide. Chem. Rev. 1998, 98, 2549-2586.

(2) Tiberg, C.; Sjöstedt, C.; Persson, I.; Gustafsson, J. P. Phosphate Effects on Copper(II) and Lead(II) Sorption to Ferrihydrite. Geochim. Cosmochim. Acta 2013, 120, 140-157.

(3) Tiberg, C.; Gustafsson, J. P. Phosphate Effects on Cadmium(II) Sorption to Ferrihydrite. J. Colloid Interface Sci. 2016, 471, 103-111.

(4) Davis, J. A.; Leckie, J. O. Surface Ionization and Complexation at the Oxide/Water Interface II. Surface Properties of Amorphous Iron Oxyhydroxide and Adsorption of Metal Ions. J. Colloid Interface Sci. 1978, 67, 90-107.

(5) Goldberg, S. Competitive Adsorption of Arsenate and Arsenite on Oxides and Clay Minerals. Soil Sci. Soc. Am. J. 2002, 66, 413-421.

(6) Hiemstra, T.; Zhao, W. Reactivity of Ferrihydrite and Ferritin in Relation to Surface Structure, Size, and Nanoparticle Formation Studied for Phosphate and Arsenate. Environ. Sci. Nano 2016, 3, $1265-1279$.

(7) Schwertmann, U. Occurrence and Formation of Iron Oxides in Various Pedoenvironments. In Iron in Soils and Clay Minerals; Stucki, J. W.; Goodman, B. A.; Schwertmann, U., Eds.; Springer: Netherlands: Dordrecht, 1988; pp 267-308.

(8) Karltun, E.; Bain, D. C.; Gustafsson, J. P.; Mannerkoski, H.; Murad, E.; Wagner, U.; Fraser, A. R.; McHardy, W. J.; Starr, M. Surface Reactivity of Poorly-Ordered Minerals in Podzol B Horizons. Geoderma 2000, 94, 265-288. 
(9) Tiberg, C.; Sjöstedt, C.; Gustafsson, J. P. Metal Sorption to Spodosol Bs Horizons: Organic Matter Complexes Predominate. Chemosphere 2018, 196, 556-565.

(10) Banzhaf, S.; Filipovic, M.; Lewis, J.; Sparrenbom, C. J.; Barthel, R. A Review of Contamination of Surface-, Ground-, and Drinking Water in Sweden by Perfluoroalkyl and Polyfluoroalkyl Substances (PFASs). Ambio 2017, 46, 335-346.

(11) Park, S.; Lee, L. S.; Medina, V. F.; Zull, A.; Waisner, S. HeatActivated Persulfate Oxidation of PFOA, 6:2 Fluorotelomer Sulfonate, and PFOS under Conditions Suitable for in-Situ Groundwater Remediation. Chemosphere 2016, 145, 376-383.

(12) von der Trenck, K. T.; Konietzka, R.; Biegel-Engler, A.; Brodsky, J.; Hädicke, A.; Quadflieg, A.; Stockerl, R.; Stahl, T. Significance Thresholds for the Assessment of Contaminated Groundwater: Perfluorinated and Polyfluorinated Chemicals. Environ. Sci. Eur. 2018, 30, No. 19.

(13) Hellsing, M. S.; Josefsson, S.; Hughes, A. V.; Ahrens, L. Sorption of Perfluoroalkyl Substances to Two Types of Minerals. Chemosphere 2016, 159, 385-391.

(14) Tang, C. Y.; Shiang, Fu. Q.; Gao, D.; Criddle, C. S.; Leckie, J. O. Effect of Solution Chemistry on the Adsorption of Perfluorooctane Sulfonate onto Mineral Surfaces. Water Res. 2010, 44, 2654-2662.

(15) Wang, F.; Shih, K. Adsorption of Perfluorooctanesulfonate (PFOS) and Perfluorooctanoate (PFOA) on Alumina: Influence of Solution PH and Cations. Water Res. 2011, 45, 2925-2930.

(16) Zhao, L.; Bian, J.; Zhang, Y.; Zhu, L.; Liu, Z. Comparison of the Sorption Behaviors and Mechanisms of Perfluorosulfonates and Perfluorocarboxylic Acids on Three Kinds of Clay Minerals. Chemosphere 2014, 114, 51-58.

(17) Gao, X.; Chorover, J. Adsorption of Perfluorooctanoic Acid and Perfluorooctanesulfonic Acid to Iron Oxide Surfaces as Studied by Flow-through ATR-FTIR Spectroscopy. Environ. Chem. 2012, 9, $148-157$.

(18) Wang, F.; Liu, C.; Shih, K. Adsorption Behavior of Perfluorooctanesulfonate (PFOS) and Perfluorooctanoate (PFOA) on Boehmite. Chemosphere 2012, 89, 1009-1014.

(19) Wang, F.; Shih, K.; Leckie, J. O. Effect of Humic Acid on the Sorption of Perfluorooctane Sulfonate (PFOS) and Perfluorobutane Sulfonate (PFBS) on Boehmite. Chemosphere 2015, 118, 213-218.

(20) Uwayezu, J.-N.; Yeung, L. W. Y.; Bäckström, M. Sorption of PFOS Isomers on Goethite as a Function of PH, Dissolved Organic Matter (Humic and Fulvic Acid) and Sulfate. Chemosphere 2019, 233, 896-904.

(21) Johnson, R. L.; Anschutz, A. J.; Smolen, J. M.; Simcik, M. F.; Penn, R. L. The Adsorption of Perfluorooctane Sulfonate onto Sand, Clay, and Iron Oxide Surfaces. J. Chem. Eng. Data 2007, 52, 11651170

(22) Jeon, J.; Kannan, K.; Lim, B. J.; An, K. G.; Kim, S. D. Effects of Salinity and Organic Matter on the Partitioning of Perfluoroalkyl Acid (PFAs) to Clay Particles. J. Environ. Monit. 2011, 13, 1803-1810.

(23) Xiao, F.; Zhang, X.; Penn, L.; Gulliver, J. S.; Simcik, M. F. Effects of Monovalent Cations on the Competitive Adsorption of Perfluoroalkyl Acids by Kaolinite: Experimental Studies and Modeling. Environ. Sci. Technol. 2011, 45, 10028-10035.

(24) Xu, T.; Ji, H.; Gu, Y.; Tong, T.; Xia, Y.; Zhang, L.; Zhao, D. Enhanced Adsorption and Photocatalytic Degradation of Perfluorooctanoic Acid in Water Using Iron (Hydr)Oxides/Carbon Sphere Composite. Chem. Eng. J. 2020, 388, No. 124230.

(25) Zhang, Y.; Zhi, Y.; Liu, J.; Ghoshal, S. Sorption of Perfluoroalkyl Acids to Fresh and Aged Nanoscale Zerovalent Iron Particles. Environ. Sci. Technol. 2018, 52, 6300-6308.

(26) Hiemstra, T.; Van Riemsdijk, W. H. A Surface Structural Model for Ferrihydrite I: Sites Related to Primary Charge, Molar Mass, and Mass Density. Geochim. Cosmochim. Acta 2009, 73, 4423-4436.

(27) Goldberg, S.; Sposito, G. A Chemical Model of Phosphate Adsorption by Soils: II. Noncalcareous Soils. Soil Sci. Soc. Am. J 1984, $48,779-783$.

(28) Xia, K.; Weesner, F.; Bleam, W. F.; Helmke, P. A.; Bloom, P. R.; Skyllberg, U. L. XANES Studies of Oxidation States of Sulfur in
Aquatic and Soil Humic Substances. Soil Sci. Soc. Am. J 1998, 62, $1240-1246$

(29) Boye, K.; Almkvist, G.; Nilsson, S. I.; Eriksen, J.; Persson, I. Quantification of Chemical Sulphur Species in Bulk Soil and Organic Sulphur Fractions by S K-Edge XANES Spectroscopy. Eur. J. Soil Sci. 2011, 62, 874-881.

(30) Majzlan, J.; Alpers, C. N.; Koch, C. B.; McCleskey, R. B.; Myneni, S. C. B.; Neil, J. M. Vibrational, X-Ray Absorption, and Mössbauer Spectra of Sulfate Minerals from the Weathered Massive Sulfide Deposit at Iron Mountain, California. Chem. Geol. 2011, 284, 296-305.

(31) Prietzel, J.; Wu, Y.; Dümig, A.; Zhou, J.; Klysubun, W. Soil Sulphur Speciation in Two Glacier Forefield Soil Chronosequences Assessed by S K-Edge XANES Spectroscopy. Eur. J. Soil Sci. 2013, 64, 260-272.

(32) Zhu, M.; Northrup, P.; Shi, C.; Billinge, S. J. L.; Sparks, D. L.; Waychunas, G. A. Structure of Sulfate Adsorption Complexes on Ferrihydrite. Environ. Sci. Technol. Lett. 2014, 1, 97-101.

(33) Gu, C.; Wang, Z.; Kubicki, J. D.; Wang, X.; Zhu, M. X-Ray Absorption Spectroscopic Quantification and Speciation Modeling of Sulfate Adsorption on Ferrihydrite Surfaces. Environ. Sci. Technol. 2016, 50, 8067-8076.

(34) Swedlund, P. J.; Webster, J. G. Adsorption and Polymerisation of Silicic Acid on Ferrihydrite, and Its Effect on Arsenic Adsorption. Water Res. 1999, 33, 3413-3422.

(35) Schwertmann, U.; Cornell, R. M. Iron Oxides in the Laboratory; Wiley Books, 2000.

(36) Gustafsson, J. P.; Persson, I.; Kleja, D. B.; van Schaik, J. W. J. Binding of Iron(III) to Organic Soils: EXAFS Spectroscopy and Chemical Equilibrium Modeling. Environ. Sci. Technol. 2007, 41, $1232-1237$

(37) Klysubun, W.; Tarawarakarn, P.; Thamsanong, N.; Amonpattaratkit, P.; Cholsuk, C.; Lapboonrueng, S.; Chaichuay, S.; Wongtepa, W. Upgrade of SLRI BL8 Beamline for XAFS Spectroscopy in a Photon Energy Range of $1-13 \mathrm{keV}$. Radiat. Phys. Chem. 2020, 175, No. 108145 .

(38) Ravel, B.; Newville, M. ATHENA, ARTEMIS, HEPHAESTUS: Data Analysis for X-Ray Absorption Spectroscopy Using IFEFFIT. J. Synchrotron Radiat. 2005, 12, 537-541.

(39) Lath, S.; Knight, E. R.; Navarro, D. A.; Kookana, R. S.; McLaughlin, M. J. Sorption of PFOA onto Different Laboratory Materials: Filter Membranes and Centrifuge Tubes. Chemosphere 2019, 222, 671-678.

(40) Sörengård, M.; Franke, V.; Tröger, R.; Ahrens, L. Losses of Poly- and Perfluoroalkyl Substances to Syringe Filter Materials. J. Chromatogr. A 2020, 1609, No. 460430.

(41) Schwarzenbach, R. P.; Gschwend, P. M.; Imboden, D. M. Environmental Organic Chemistry; John Wiley \& Sons, 2016.

(42) Sharom, M. S.; Solomon, K. R. Adsorption and Desorption of Permethrin and Other Pesticides on Glass and Plastic Materials Used in Bioassay Procedures. Can. J. Fish Aquat. Sci. Can. 1981, 199-204.

(43) Chlebowski, A. C.; Tanguay, R. L.; Simonich, S. L. M. Quantitation and Prediction of Sorptive Losses during Toxicity Testing of Polycyclic Aromatic Hydrocarbon (PAH) and Nitrated PAH (NPAH) Using Polystyrene 96-Well Plates. Neurotoxicol. Teratol. 2016, 57, 30-38.

(44) Waldo, G.; Carlson, R. M. K.; Moldowan, J. M.; Peters, K. E.; Penner-Hahn, J. E. Sulfur Speciation in Heavy Petroleums: Information from X-Ray Absorption near-Edge Structure. Geochim. Cosmochim. Acta 1991, 801-814.

(45) Waldo, G. S.; Mullins, O. C.; Penner-Hahn, J. E.; Cramer, S. P. Determination of the Chemical Environment of Sulphur in Petroleum Asphaltenes by X-Ray Absorption Spectroscopy. Fuel 1992, 71, 5357.

(46) Spiro, C. L.; Wong, J.; Lytle, F. W.; Greegor, R. B.; Maylotte, D. H.; Lamson, S. H. X-Ray Absorption Spectroscopic Investigation of Sulfur Sites in Coal: Organic Sulfur Identification. Science 1984, 226, $48-50$. 
(47) Huffman, G. P.; Shah, N.; Huggins, F. E.; Stock, L. M.; Chatterjee, K.; Kilbane, J. J.; Chou, M.-I. M.; Buchanan, D. H. Sulfur Speciation of Desulfurized Coals by XANES Spectroscopy. Fuel 1995, $74,549-555$.

(48) Vairavamurthy, A.; Manowitz, B.; Luther, G. W.; Jeon, Y. Oxidation State of Sulfur in Thiosulfate and Implications for Anaerobic Energy Metabolism. Geochim. Cosmochim. Acta 1993, 57, $1619-1623$.

(49) Vairavamurthy, A.; Zhou, W.; Eglinton, T.; Manowitz, B. Sulfonates: A Novel Class of Organic Sulfur Compounds in Marine Sediments. Geochim. Cosmochim. Acta 1994, 58, 4681-4687.

(50) Okude, N.; Nagoshi, M.; Noro, H.; Baba, Y.; Yamamoto, H.; Sasaki, T. A. P and S K-Edge XANES of Transition-Metal Phosphates and Sulfates. J. Electron Spectrosc. Relat. Phenom. 1999, 101-103, 607-610.

(51) Majzlan, J.; Myneni, S. C. B. Speciation of Iron and Sulfate in Acid Waters: Aqueous Clusters to Mineral Precipitates. Environ. Sci. Technol. 2005, 39, 188-194.

(52) Almkvist, G.; Boye, K.; Persson, I. K-Edge XANES Analysis of Sulfur Compounds: An Investigation of the Relative Intensities Using Internal Calibration. J. Synchrotron Radiat. 2010, 17, 683-688.

(53) Erkoç, Ş.; Erkoç, F. Structural and Electronic Properties of PFOS and LiPFOS. J. Mol. Struct.: THEOCHEM 2001, 549, 289293.

(54) Rayne, S.; Forest, K. A New Class of Perfluorinated Acid Contaminants: Primary and Secondary Substituted Perfluoroalkyl Sulfonamides Are Acidic at Environmentally and Toxicologically Relevant PH Values. J. Environ. Sci. Health, Part A: Toxic/Hazard. Subst. Environ. Eng. 2009, 44, 1388-1399.

(55) Steinle-Darling, E.; Reinhard, M. Nanofiltration for Trace Organic Contaminant Removal: Structure, Solution, and Membrane Fouling Effects on the Rejection of Perfluorochemicals. Environ. Sci. Technol. 2008, 42, 5292-5297.

(56) Tunega, D.; Gerzabek, M. H.; Haberhauer, G.; Totsche, K. U.; Lischka, H. Model Study on Sorption of Polycyclic Aromatic Hydrocarbons to Goethite. J. Colloid Interface Sci. 2009, 330, 244249.

(57) Lu, X.; Deng, S.; Wang, B.; Huang, J.; Wang, Y.; Yu, G. Adsorption Behavior and Mechanism of Perfluorooctane Sulfonate on Nanosized Inorganic Oxides. J. Colloid Interface Sci. 2016, 474, 199205.

(58) Rayne, S.; Forest, K. Perfluoroalkyl Sulfonic and Carboxylic Acids: A Critical Review of Physicochemical Properties, Levels and Patterns in Waters and Wastewaters, and Treatment Methods. J. Environ. Sci. Health, Part A: Toxic/Hazard. Subst. Environ. Eng. 2009, $44,1145-1199$.

(59) Sørli, J. B.; Låg, M.; Ekeren, L.; Perez-Gil, J.; Haug, L. S.; Da Silva, E.; Matrod, M. N.; Gützkow, K. B.; Lindeman, B. Per- and Polyfluoroalkyl Substances (PFASs) Modify Lung Surfactant Function and pro-Inflammatory Responses in Human Bronchial Epithelial Cells. Toxicol. In Vitro 2020, 62, No. 104656.

(60) Shinoda, K.; Hato, M.; Hayashi, T. Physicochemical Properties of Aqueous Solutions of Fluorinated Surfactants. J. Phys. Chem. A. 1972, 76, 909-914.

(61) Kissa, E. Fluorinated Surfactants and Repellents; Marcel Dekker, 2001.

(62) Brusseau, M. L. Estimating the Relative Magnitudes of Adsorption to Solid-Water and Air/Oil-Water Interfaces for perand Poly-Fluoroalkyl Substances. Environ. Pollut. 2019, 254, No. 113102

(63) Guelfo, J. L.; Higgins, C. P. Subsurface Transport Potential of Perfluoroalkyl Acids at Aqueous Film-Forming Foam (AFFF)Impacted Sites. Environ. Sci. Technol. 2013, 47, 4164-4171.

(64) Higgins, C. P.; Luthy, R. G. Sorption of Perfluorinated Surfactants on Sediments. Environ. Sci. Technol. 2006, 40, 7251-7256.

(65) Chen, Y.-C.; Lo, S.-L.; Li, N.-H.; Lee, Y.-C.; Kuo, J. Sorption of Perfluoroalkyl Substances (PFASs) onto Wetland Soils. Desalination Water Treat. 2013, 51, 7469-7475.
(66) Milinovic, J.; Lacorte, S.; Vidal, M.; Rigol, A. Sorption Behaviour of Perfluoroalkyl Substances in Soils. Sci. Total Environ. 2015, 511, 63-71.

(67) Zhao, L.; Zhang, Y.; Fang, S.; Zhu, L.; Liu, Z. Comparative Sorption and Desorption Behaviors of PFHxS and PFOS on Sequentially Extracted Humic Substances. J. Environ. Sci. 2014, 26, 2517-2525.

(68) Zhi, Y.; Liu, J. Sorption and Desorption of Anionic, Cationic and Zwitterionic Polyfluoroalkyl Substances by Soil Organic Matter and Pyrogenic Carbonaceous Materials. Chem. Eng. J. 2018, 346, 682-691.

(69) Schwertmann, U.; Fechter, H. The Point of Zero Charge of Natural and Synthetic Ferrihydrites and Its Relation to Adsorbed Silicate. Clay Miner. 1982, 471-476.

(70) Rhoton, F. E.; Bigham, J. M. Natural Ferrihydrite as an Agent for Reducing Turbidity Caused by Suspended Clays. J. Environ. Qual. 2009, 38, 1887-1891.

(71) Oliver, D. P.; Li, Y.; Orr, R.; Nelson, P.; Barnes, M.; McLaughlin, M. J.; Kookana, R. S. The Role of Surface Charge and PH Changes in Tropical Soils on Sorption Behaviour of Per- and Polyfluoroalkyl Substances (PFASs). Sci. Total Environ. 2019, 673, 197-206. 